\title{
High frequency oscillations in the solar chromosphere and their connection with heating
}

\author{
Aleksandra Andic ${ }^{1}$, M. Mathioudakis ${ }^{1}$, F. P. Keenan ${ }^{1}$ D. B. Jess ${ }^{1,2}$ \\ and D. S. Bloomfield ${ }^{3}$ \\ ${ }^{1}$ Queen's University Belfast \\ University Road, Belfast, BT7 1NN, UK \\ email: a.andic@qub.ac.uk \\ ${ }^{2}$ NASA Goddard Space Flight Center, Soar Physics Laboratory \\ Code 612, Greenbelt, MD 20771 USA \\ ${ }^{3}$ Max Planck Institut fuer Sonnensystemforshung \\ Max-Planck-Str.2, 37191 Katlenburg-Lindau, Germany
}

\begin{abstract}
High frequency acoustic waves have been suggested as a source of mechanical heating in the quiet solar chromosphere. To investigate this, we have observed intensity oscillations of several lines in the frequency interval $1.64-70 \mathrm{mHz}$ using data from the VTT Tenerife and the Dunn Solar Telescope at the National Solar Observatory. Our analysis of Fe I $543.45 \mathrm{~nm}$, Fe I $543.29 \mathrm{~nm}$ and the G-band, indicate that the majority of oscillations are connected with the magnetic fields and do not provide sufficient mechanical flux for the heating of the chromosphere. This correlation is also observed in quiet Sun areas.
\end{abstract}

Keywords. oscillations, heating, chromosphere

\section{Introduction}

For many years, high frequency acoustic waves have been suggested as a source of mechanical heating in the solar atmosphere. The majority of the models which offer this explanation employ a 1-dimensional approximation. Ulmschneider (1971a, 1971b, 2003) suggested that waves with frequencies from $6 \mathrm{mHz}$ to $100 \mathrm{mHz}$ are the main carrier of the required energy, and the peak energy transport should occur for waves with frequencies greater than $20 \mathrm{mHz}$. It is thought that the existence of the chromosphere depends on a time-constant energy supply provided by mechanical heating (Ulmscheider 2003).

High frequency oscillations have been observed and analyzed since this explanation was first suggested (Liu, 1974; Mein \& Mein, 1976; von Uexküll et al., 1985; Hansteen, Betta \& Carlsson, 2000; Wunnenberg, Andjic \& Kneer, 2003; Andic, 2007a). The observed oscillations, and energy they carry, have been discussed by several authors (Liu, 1974; Lites \& Chipman, 1979; Fossum \& Carlsson, 2006; Andic, 2007a ). All of these conclude that the observed energy is insufficient to account for the chromospheric losses.

The origin of the oscillations is still not clear (Moretti et al. 2001). It is believed that the majority of acoustic waves originate from isolated sources which occupy relatively small volumes. However, the nature of those sources has not been determined (Deubner \& Laufer 1983). Also, some research (Domìnguez, 2004; Andjic \& Wiehr, 2006) provides evidence for magnetic activity even in the so-called "non-magnetic" internetwork. From theoretical standpoint there are evidences that magnetic field channels and modulate high frequency oscillations (James et al.,2003; Erdélyi et al. 2007). This questions the assumption that the observed high oscillations are purely acoustic in origin. 


\section{Data}

The datasets used in the present analysis were obtained using two ground-based telescopes. The first dataset was obtained with the Dunn Solar Telescope (DST) at Sacramento Peak. Data in the G-band were taken at a cadence of $0.05 \mathrm{~s}$ using the Rapid Dual Imager (RDI). Details of this instrument and the observations may be found in Jess et al. (2007).

The second dataset, which includes observations of the Fe I $543.45 \mathrm{~nm}$ and $543.29 \mathrm{~nm}$ lines, was obtained using the German Vacuum Tower telescope (VTT) on the Canary islands, with the "Göttingen" Fabry-Perot spectrometer (Bendlin, Volkmer \& Kneer 1992). Details of these observations may be found in Andic (2007).

\section{Analysis}

All datasets were subject to the speckle reconstruction of Andic (2007a), and the detection of oscillations was performed using wavelet analysis. Data from the VTT were also subject to a position analysis as discussed by Andic (2007b). Line weakening may appear in the presence of the magnetic field. To establish the locations where this occurs, the determination of line weakening was undertaken as described in Stellmacher $\&$ Wiehr (1979). For the purposes of our analysis, it is assumed that, although the core of Fe I I $543.45 \mathrm{~nm}$ is formed around the temperature minimum, non-LTE uniformly affects all the lines employed, i.e. the formation layer of the line core does not vary much in height between granulation and intergranular lanes. An intensity parameter for the possible variation of the atmosphere affected by the waves is the referent line profile, i.e. normalized to the corresponding continuum: $I_{\lambda}^{\text {rel }}=I_{\lambda} I_{\text {cont }}^{-1}$.

The method is applied on the whole field-of-view for the VTT dataset. As for the relative line profile, the average profile is calculated over areas where velocity maps shows the existence of the up-flow. This increases the probability that the relevant profile is obtained from non-magnetic areas. Both profiles are normalized to the continuum point, which is the last point in the line scan. Line weakening is a sufficient but not necessary condition for the existence of the magnetic flux concentrations, i.e. every flux tube has a "weakening", but not every weakening must indicate a flux concentration.

The phase difference is calculated for the VTT datasets, and the phase difference spectrum is formed such that an upward propagating wave leads to positive phase differences. Phase differences are presented in a weighted diagram, where weighting is applied per sample by cross-power amplitude $\sqrt{P_{1} P_{2}}$ to produce binned greyscale phase, $\Delta \phi$, displayed with normalization per temporal frequency bin (Lites \& Chipman 1979)

\section{Results}

\section{1. $R D I$}

Oscillations were observed within a frequency range of $1 \mathrm{mHz}$ to $125 \mathrm{mHz}$. They appear with various durations, the most common being two cycles, i.e. relatively short bursts ( Fig. 1). The oscillations were usually located above areas where the magnetic flux concentrations are expected, i.e. edge of the umbra, penumbra and bright points along the rest of the field-of-view.

Oscillations of different frequencies appear in the same spatial locations and at the same times. This could indicate that the sources of the very high frequency oscillations are the same as those for oscillations at other frequencies. 


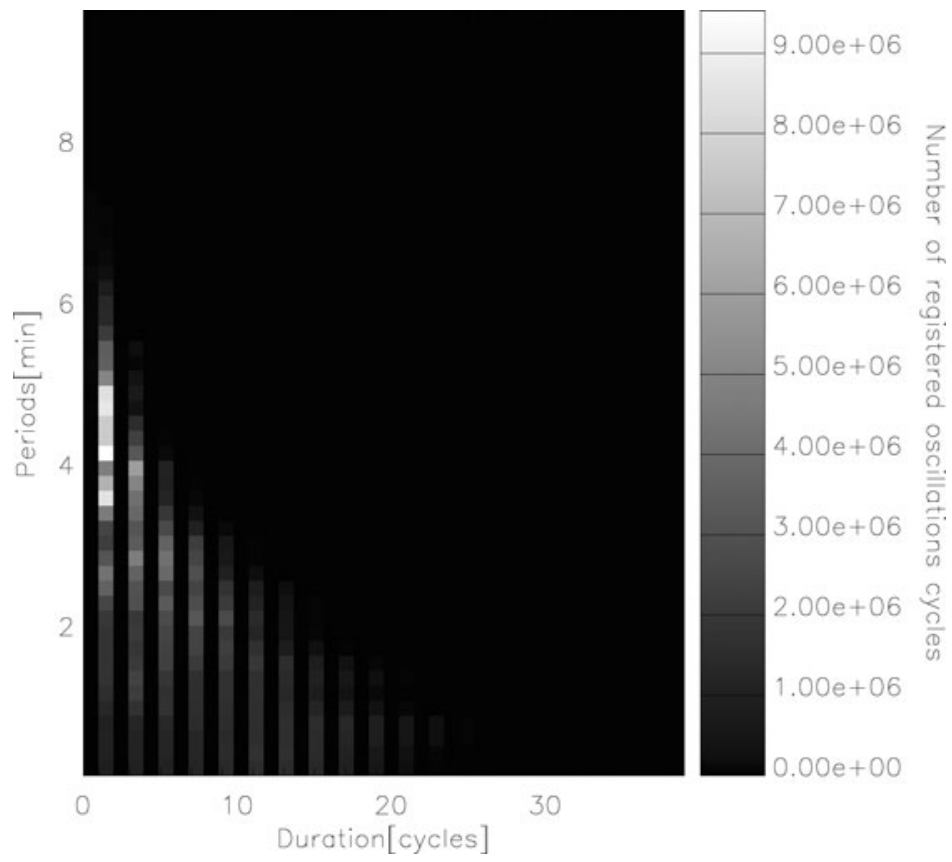

Figure 1. Duration of the oscillations observed with the RDI instrument.

\section{2. $V T T$}

Here, we observed oscillations in the frequency range $0.8 \mathrm{mHz}$ to $22 \mathrm{mHz}$. Their duration showed a similar behavior to those observed with the RDI. However, a tendency towards shorter bursts was even more prominent. Oscillations did not show a definite preference for a location, and were observed to be almost equally distributed between granules and intergranular lanes (Andic 2007b). A significant percentage (around 70\%) was located in the areas where the line weakening was registered.
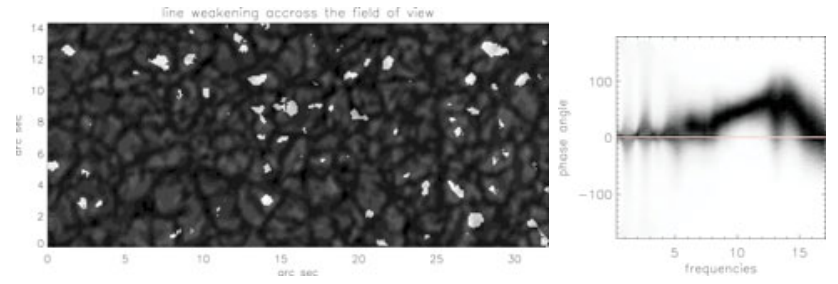

Figure 2. The first panel represents the location of the line weakening across the field-of-view, while the second represents the phase difference calculated for the VTT data.

Oscillations which were observed at the location of the line weakening are propagating upwards. The second panel in Fig. 2 shows the phase difference calculated for the line curves from the whole field-of-view. The positive difference marks the oscillations traveling upward.

\section{Discussion and Conclusions}

Although it is believed that acoustic oscillations are responsible for the heating of the chromosphere, the most recent observations (Fossum \& Carlsson, 2007; Andic, 2007a) 
show that mechanical oscillations do not carry sufficient energy. Also, recently there have been several observational and theoretical studies (Domìnguez, 2004; Andjic \& Wiehr, 2006; Trujillo Bueno, Shuckina \& Asensio Ramos 2004) which state that the magnetic field strength in the quiet Sun is much larger than previously thought. This, together with the fact that we find in the present work that most of the oscillations appear in the areas where magnetic flux concentrations are expected, leads us to conclude that the role of the magnetic field is much larger in the generation of the observed high-energy oscillations. Therefore, heating of the chromosphere cannot be supplied primarily via mechanical oscillations.

\section{Acknowledgments}

AA is grateful to the Science and Technology Facilities Council for financial support, while DBJ acknowledges support both from the Northern Ireland Department of Education and Learning, and NASA Goddard Space Flight Center. FPK is grateful to AWE for the award of a William Penney Fellowship.

\section{References}

Andic, A. 2007 Solar Phys.,242,9A

Andic, A. 2007 Solar Phys.,tmp,94A

Andjic, A. \& Wiehr, E. 2006, Publ. Astron. Obs. Belgrade, 80, 367

Bendlin, C., Volkmer, R., Kneer, F. 1992, A \& A, 257, 817

Deubner, F.L. \& Laufer, J., 1983, Solar Phys., 82, 151

Domìnguez, I.F. 2004, Quiet Sun Magnetic Fields, Copernikus GmbH, Katlenburg-Lindau, Germany, p.111

Erdélyi, R., Malins, C., Tóth, G., \& De Pontieu, B. 2007, A \& A, 467, 1299

Fossum, A. \& Carlsson, M. 2006, ApJ, 646, 579

Hansteen, V.H., Betta, R., Carlsson, M. 2000, A $\mathscr{E}$ A, 360, 742

James, S.P., Erdélyi, R. \& De Pontieu, B. 2003, A \& A, 406, 715

Jess, D.B., Andic, A., Mathioudakis, M., Bloomfield, D.S., \& Keenan, F.P. 2007, A $\&$ A, 473, 943

Lites, B.W. \& Chipman, E.G. 1979, ApJ, 231, 570-588

Liu, S.Y. 1974, ApJ, 189, 359

Mein,N. \& Mein,P. 1976, Solar Phys., 49, 231

Moretti, P.F., Cacciani, A., Hanslmeier, A., Messerotti, M., Oliviero, M., Otruba, W., Severino, G., Warmuth, A. 2001, $A$ \& $A, 372,1038$

Stellmacher, G. \& Wiehr, E. 1979, A \& $A$, 75, 263-267

Trujillo Bueno, J., Shchukina, N., Asensio Ramos, A. 2004, Nature, 430, 326

Ulmschneider, P. 1971a, $A$ \& $A$, 12, 297

Ulmschneider, P. 1971b, $A$ \& $A, 14,275$

Ulmschneider, P. 2003, In: Antia, H.M., Bhatnagar, A., Ulmschneider,P. (eds.), Lectures on solar Physic, Lecture Notes in Physics, Vol.619, Springer Verlag, Berlin, p.232

von Uexküll, M., Kneer, F., Mattig, W., Nesis, A., Schmidt, W. 1985, A \& A A, 146, 192

Wunnenberg, M., Andjic, A., Kneer, F. 2003, Astron. Nachr., 324, 356 